\title{
Role for Subthalamic Nucleus Neurons in Switching from Automatic to Controlled Eye Movement
}

\author{
Masaki Isoda ${ }^{1,2}$ and Okihide Hikosaka ${ }^{1}$ \\ ${ }^{1}$ Laboratory of Sensorimotor Research, National Eye Institute, National Institutes of Health, Bethesda, Maryland 20892, and ${ }^{2}$ Laboratory for Symbolic \\ Cognitive Development, RIKEN Brain Science Institute, Wako, Saitama 351-0198, Japan
}

The subthalamic nucleus (STN) of the basal ganglia is an important element of motor control. This is demonstrated by involuntary movements induced by STN lesions and the successful treatment of Parkinson's disease by STN stimulation. However, it is still unclear how individual STN neurons participate in motor control. Here, we report that the STN has a function in switching from automatic to volitionally controlled eye movement. In the STN of trained macaque monkeys, we found neurons that showed a phasic change in activity specifically before volitionally controlled saccades which were switched from automatic saccades. A majority of switch-related neurons were considered to inhibit no-longer-valid automatic processes, and the inhibition started early enough to enable the animal to switch. We suggest that the STN mediates the control signal originated from the medial frontal cortex and implements the behavioral switching function using its connections with other basal ganglia nuclei and the superior colliculus.

Key words: subthalamic nucleus; basal ganglia; monkeys; saccades; habitual action; conscious control

\section{Introduction}

The subthalamic nucleus (STN) of the basal ganglia plays an important role in motor control. This concept has been supported by ample evidence derived mainly from clinicopathological studies. Specifically, lesions within the STN produce ballistic involuntary movements of the contralateral extremities (Crossman et al., 1984). Increased activity in the STN is postulated to underlie akinetic motor symptoms of Parkinson's disease (Bergman et al., 1990, 1994). Deep brain stimulation or lesioning of the parkinsonian STN results in a marked improvement in motor function (Bergman et al., 1990; Limousin et al., 1995). Finally, disruption of the STN causes impulsive responding (Baunez et al., 1995; Frank et al., 2007). These observations, together with the basal ganglia network models (DeLong, 1990; Mink, 1996; Nambu et al., 2002) (supplemental Fig. 1, available at www. jneurosci.org as supplemental material), suggest that the STN plays a role in motor response inhibition. However, there are few studies in intact subjects that have tested this hypothesis, except a functional neuroimaging study recently conducted in humans (Aron and Poldrack, 2006). Physiological evidence has yet to be provided at the single-cell level.

A unique feature of the STN among basal ganglia nuclei is that it receives inputs directly from the frontal cortex, bypassing path-

Received Feb. 3, 2008; revised May 7, 2008; accepted June 4, 2008.

This work was supported by the intramural research program of the National Eye Institute. We thank R. H. Wurtz, K. Nakamura, L. Ding, M. Matsumoto, S. Hong, and E. Bromberg-Martin for comments and discussions; B. G. Cumming for data analysis; M. K. Smith for histological expertise; and J. W. McClurkin, T. W. Ruffner, A. M. Nichols, A. V. Hays, and L. P. Jensen for technical assistance.

Correspondence should be addressed to Dr. Masaki Isoda, Laboratory for Symbolic Cognitive Development, RIKEN Brain Science Institute, 2-1 Hirosawa, Wako, Saitama 351-0198, Japan. E-mail: isodam@brain.riken.jp. DOI:10.1523/JNEUROSCI.0487-08.2008

Copyright $\odot 2008$ Society for Neuroscience $\quad$ 0270-6474/08/287209-10\$15.00/0 ways involving the striatum (Monakow et al., 1978; Nambu et al., 1996), and that this hyperdirect fronto-subthalamic pathway is fast conducting (Nambu et al., 2000) compared with much slower striatal pathways (Yoshida and Precht, 1971; Bauswein et al., 1989; Hikosaka et al., 1993). This raises the possibility that the STN is suitable for quick control of action. One situation that requires quick control is switching of behavior, particularly when a sudden change in the environment requires a cessation of automatic ongoing activity and instead deliberately controlled behavior must be performed (Norman and Shallice, 1986). Several studies show that the medial frontal cortex, especially the presupplementary motor area (pre-SMA), plays a key role in such a behavioral switching process (Shima et al., 1996; Nakamura et al., 1998; Rushworth et al., 2002; Nachev et al., 2005, 2007; Crone et al., 2006; Woodward et al., 2006; Isoda and Hikosaka, 2007). However, it remains unanswered how the pre-SMA achieves its switching function in the motor system. A plausible hypothesis is that the pre-SMA switch signal is implemented by the STN, through direct connections from the pre-SMA to the STN (Inase et al., 1999). Indeed, the STN has been suggested to play a role in the switching of movement (Monchi et al., 2006), yet the detailed underlying mechanism is unknown.

Here, we test our hypothesis at the single-cell level: the STN is centrally concerned with inhibiting habitual automatic actions when a novel behavioral context requires a controlled alternative action. We show that a group of STN neurons indeed was capable of switching from automatic to controlled action. We also show that the majority of these neurons appear to inhibit automatic inappropriate action but others were related to facilitation of controlled desired action. We discuss possible neuronal networks through which the STN might exert its switching functions. 


\section{Materials and Methods}

We used two rhesus monkeys (Macaca mulatta), T and S, in this study. All animal care and experimental procedures were approved by the Institute Animal Care and Use Committee and complied with the Public Health Service Policy on the humane care and use of laboratory animals. Surgical procedures. The monkey was anesthetized with intramuscular injections of ketamine $\mathrm{HCl}(10 \mathrm{mg} / \mathrm{kg})$, diazepam $(1 \mathrm{mg} / \mathrm{kg})$, and glycopyrrolate $(0.01 \mathrm{mg} / \mathrm{kg})$, and then maintained at a general anesthetized state with isofluorane. After the skull was exposed, acrylic screws were installed to fasten the dental acrylic head implant to the skull. A plastic head holder and recording chambers were placed stereotaxically and secured with dental acrylic. Eye coils were implanted subconjunctivally into both eyes using the methods described previously (Judge et al., 1980). Craniotomy was performed after the monkey had been well trained for the behavioral tasks described below. Antibiotics and analgesics were administered after surgery.

Behavioral tasks. During experimental sessions, the monkey was placed in a sound-attenuated room and seated in a primate chair with its head immobilized. Behavioral tasks were under control of a QNX-based real-time experimentation data acquisition system [REX; Laboratory of Sensorimotor Research, National Eye Institute, National Institutes of Health (LSR/NEI/NIH)]. Visual stimuli were rear-projected by an active matrix liquid crystal display projector (PJ550; ViewSonic) onto a frontoparallel screen $33 \mathrm{~cm}$ from the monkey's eyes. Water reward was delivered after a monkey's correct response through a spigot under the control of a solenoid valve, which was placed outside the sound-attenuated room. The monkey was trained to perform several oculomotor tasks using operant conditioning with positive reinforcement: a memoryguided saccade task, saccade overriding task, and saccade go/no-go task. All trials started with the presentation of a white spot of light [fixation point $(\mathrm{FP})]$ at the center of the screen on which the monkeys had to fixate.

The memory-guided saccade task was used to estimate the response field (RF) of a cell under study. In this task, a peripheral cue appeared transiently for $100 \mathrm{~ms}$ after the monkey had fixated on the FP for $800 \mathrm{~ms}$. The monkey was required to remember the cued location while maintaining fixation on the FP for another $800-1200 \mathrm{~ms}$. The FP then went off and the monkey had to make a saccade to the cued location.

The saccade overriding task (see Fig. $1 A$ ) was designed to investigate control over automatic responses. In this task, after the monkey had fixated on the FP for $1 \mathrm{~s}$, two colored stimuli (yellow and pink) appeared in the periphery. The positions of the stimuli were randomly determined out of two possible locations: one in the RF of the cell and the other in the diametrically opposite position if a cell under study had the clear RF; otherwise the stimuli were presented to the right and to the left of center $\left(10-20^{\circ}\right.$ in eccentricity). After a short delay $(200 \mathrm{~ms}$ for monkey $\mathrm{T}, 100$ ms for monkey S), the white FP changed to one of the two stimulus colors as a cue. The monkey was then required to make a saccade within $1 \mathrm{~s}$ to the stimulus whose color was the same as the cue (target stimulus). A reward was given after the monkey captured the target stimulus and maintained the fixation for another $300 \mathrm{~ms}$. When the monkey made a saccade to a nontarget stimulus, a tone signal was given for $200 \mathrm{~ms}$ as an error feedback as soon as the monkey's direction of gaze entered an electronic window centered at the wrong target, which was then followed by extinction of all the visual stimuli. Importantly, the color of the cue remained unchanged during a block of trials (the number of trials per block varied randomly: $1-10$ for monkey T; $1-8$ for monkey $S$ ) and then switched in the next block. We refer to the first trials of individual blocks as "switch trials" and the remaining trials as "nonswitch trials." The interval between the stimulus onset and cue onset was shorter for monkey S ( $100 \mathrm{~ms})$ than for monkey $\mathrm{T}(200 \mathrm{~ms})$ because monkey S was unable to succeed in the switch trials when the interval was equal to, or longer than, $150 \mathrm{~ms}$ (percentage of error, 93.1\%) because of very quick responses occurring in the nonswitch trials [mean saccadic reaction time (SRT), $116.6 \mathrm{~ms}$; median SRT, $98.0 \mathrm{~ms}$ ].

The saccade go/no-go task (see Fig. $1 B$ ) was devised to examine whether individual switch neurons were related to facilitation of saccades or suppression of saccades. In this task, after the monkey had fixated on the FP for $1 \mathrm{~s}$, a peripheral colored stimulus (either yellow or pink) appeared randomly at one of two possible locations: either in the RF of the cell or in the diametrically opposite position to it if a cell under study had the clear RF; otherwise the stimulus was presented to the right or to the left of center $\left(10-20^{\circ}\right.$ in eccentricity). After a short delay (200 ms for monkey T, $50 \mathrm{~ms}$ for monkey S), the white FP changed to either yellow or pink. If the colors of the FP and the stimulus were the same (50\% of all trials), the monkey had to make a saccade to the stimulus within $400 \mathrm{~ms}$ (go trial). If their colors were different, the monkey had to withhold a saccade to the stimulus and instead continue to fixate for $600 \mathrm{~ms}$ (no-go trials). A reward was given after correct go and no-go responses.

Recording procedures. Eye movements were recorded with the use of the magnetic search-coil technique with temporal resolution of $1 \mathrm{~ms}$ (Robinson, 1963). Single-unit recordings were performed using tungsten electrodes with impedances of 1.5-3 M $\Omega$ (Frederick Haer). The electrode was driven by a hydraulic micromanipulator (MO-97A; Narishige) through a stainless-steel guide tube, which was used to penetrate the dura and was held in place by a grid that was fixed inside the recording chamber (Crist et al., 1988). This grid system allowed the positioning of the electrodes at every $1 \mathrm{~mm}$ between penetrations. Neuronal activity was amplified, bandpass filtered $(600 \mathrm{~Hz}$ to $8 \mathrm{kHz})$, and single units were isolated using custom voltage-time window discrimination software (MEX; LSR/NEI/NIH). We sampled all well-isolated single neurons that potentially had visuo-oculomotor activity.

Localization of the STN. The recording chamber was tilted $38^{\circ}$ laterally in the coronal plane, with its center aimed stereotaxically at anterior 14 $\mathrm{mm}$, lateral $5 \mathrm{~mm}$, and horizontal $10 \mathrm{~mm}$ on the Horsely-Clark stereotaxic coordinate (Kusama and Mabuchi, 1970). To determine the location of the STN, we obtained magnetic resonance images of the brain before unit recording ( 3 tesla; Philips). The magnetic resonance images provided the approximate location of the STN in relation to the $x y z$ coordinate of the chamber.

The discharge of neurons in the STN was usually irregular and sometimes occurred in doublets or triplets, giving a characteristic bursting quality. The background discharge rate of STN neurons, which was measured during a $500 \mathrm{~ms}$ epoch preceding the onset of FP, was $28.3 \pm 13.0$ spikes/s (mean $\pm \mathrm{SD}, n=145$ ). These firing properties of STN neurons were in accordance with previous reports (DeLong et al., 1985; Matsumura et al., 1992; Wichmann et al., 1994).

Histology. Several marking lesions were made in and around the STN of monkey $\mathrm{T}$ by passing DCs through the recording electrodes $(10 \mu \mathrm{A}$, $35-40 \mathrm{~s}, 350-400 \mu \mathrm{C}$ ). At the end of the experiments, the monkey was deeply anesthetized with an overdose of pentobarbital sodium, and was perfused transcardially with saline followed by $4 \%$ formaldehyde. The head then was fixed to the stereotaxic device, and the brain was cut into blocks in the coronal plane parallel to the electrode penetrations. Frozen sections were cut every $50 \mu \mathrm{m}$ using a microtome and were stained for Nissl using thionin.

Data analysis. To define a switch neuron, the firing rate during a presaccadic interval in the saccade overriding task was compared among the following three trial conditions: case 1, correct switch trials in which the saccade was directed to the target in one direction; case 2, correct nonswitch trials in which the saccade was directed to the same target as in case 1; case 3, correct nonswitch trials in which the saccade was directed to the target opposite to case 1.

A neuron was then accepted as a switch neuron if the firing rate during the presaccadic interval (see below) in case 1 was significantly higher (for the increase type) or lower (for the decrease type) than that in both case 2 and case 3 (one-way ANOVA, $p<0.05$, followed by Tukey's test, $p<$ $0.05)$. We performed this procedure separately for the contralateral target and ipsilateral target. To determine the presaccadic interval for the above comparison, we first analyzed all of the data using time windows ranging from 50 to $100 \mathrm{~ms}$ duration before saccade initiation with a $5 \mathrm{~ms}$ step. We then chose a $70 \mathrm{~ms}$ window as the presaccadic interval. Our choice was justified by the fact that the ensemble-averaged activity in the switch trials aligned with saccade onset diverged from that in the nonswitch trials at 76 and $56 \mathrm{~ms}$ before saccade onset for increase-type switch neurons and decrease-type switch neurons, respectively (data not shown). 
We considered case 3 in the above comparison to rule out a spurious effect derived from the difference in the monkey's set in the initial phase of the switch trials. Suppose that a neuron simply displays directionselective activity before contralateral saccades without any additional switch-related activity. This neuron would not fire before ipsilateral saccades in the nonswitch trials (case 2). When the target was presented ipsilaterally on the switch trial (case 1), this neuron would initially fire because the monkey had been primed for the contralateral nontarget (because it had been the target). Although the monkey eventually switched correctly to the ipsilateral saccade, the neuronal activity driven by the primed set may have remained until the onset of the correct ipsilateral saccade. The comparison between case 1 and case 2 alone would characterize this neuron as switch-related. The neuron, however, would fire before contralateral saccades in the nonswitch trials (case 3) as well as in case 1 . The inclusion of case 3 would thus eliminate such direction-selective activity.

Continuous neuronal activation functions [spike density functions (SDFs) ] were generated by convolving each spike with a Gaussian kernel (SD, $10 \mathrm{~ms}$ ). Ensemble-averaged SDFs were then constructed by averaging individual SDFs with weights depending on the number of trials for each neuron.

To measure the behavioral differentiation time in the saccade overriding task (see Fig. 2 B), the frequency distribution of SRTs was constructed for both correct switch trials and incorrect switch trials (bin width, $5 \mathrm{~ms}$ ). The binomial test $(p<0.05)$ was then applied to each bin to test whether the occurrence of correct switch trials was significantly larger than chance. The behavioral differentiation time was determined at the center of the first bin from which a significant difference continued for at least 10 bins (i.e., $50 \mathrm{~ms}$ ).

Characterization of the function of individual neurons in the saccade go/no-go task was performed based on the following logic: if a neuron is related to a saccade generation process (go type), the peak activity of that neuron during the presaccadic interval in the correct go trials must exceed the peak activity in the correct no-go trials. In contrast, if a neuron is related to a saccade suppression process (no-go type), the peak activity in the correct no-go trials must exceed the peak activity of that neuron during the presaccadic interval in the correct go trials. To this end, we compared the peak values of SDF in the following time windows between go and no-go trials: for go trials, we aligned the neuronal activity with saccade onset and set a $70 \mathrm{~ms}$ window before saccade onset (we used the same duration of time window as in the saccade overriding task). For no-go trials, we aligned the neuronal activity with cue onset and set a $70 \mathrm{~ms}$ window whose center was at the behavioral differentiation time for the saccade go/ no-go task (see below). The latter choice was based on the assumption that, for the monkey to reliably cancel the saccade in no-go trials, the necessary inhibitory process must have finished by the behavioral differentiation time. To determine the statistical significance of difference in the peak SDF values between go and no-go trials, we used a resampling procedure $(n=1000)$ to estimate a $95 \%$ confidence interval of the activity difference (Efron and Tibshirani, 1993).

The behavioral differentiation time for the saccade go/no-go task was measured in an analogous manner to that in the saccade overriding task. In short, the frequency distribution of SRTs (bin width, $5 \mathrm{~ms}$ ) was constructed for the correct go trials and incorrect no-go trials (equal to the noncancellation error). The binomial test $(p<0.05)$ was then applied to each bin to test whether the occurrence of correct no-go trials was significantly higher than chance. The behavioral differentiation time was determined at the center of the first bin from which a significant difference continued consecutively for at least 10 bins (i.e., $50 \mathrm{~ms}$ ), which was 188 $\mathrm{ms}$ for monkey $\mathrm{T}$ and $223 \mathrm{~ms}$ for monkey S.

The neuronal differentiation time for individual switch neurons (see Fig. $5 B$ ) was computed using resampling (Efron and Tibshirani, 1993). Suppose we had 15 correct switch trials and 20 correct nonswitch trials for a particular neuron. For this neuron, we drew 15 trials with replacement at random from the switch trials and constructed SDF aligned on the cue onset. Likewise, we drew 20 trials with replacement at random from the nonswitch trials and constructed SDF aligned on the cue onset. We then sought the time at which the two SDFs started to differentiate. For this purpose, we first searched for the time bin (bin width, $1 \mathrm{~ms}$ ) where the difference in the two SDFs was maximum between the cue onset and the longest SRT in that session. We then sought backward in time for the first bin where the two SDFs first crossed ("intersection time"). To estimate the confidence limits for the intersection time, we repeated this procedure 1000 times and took the 97.5th percentile point as the neuronal differentiation time for this particular neuron. For the comparison of SDFs between the switch trials and nonswitch trials, the saccade target in the switch trials was in the opposite direction of the saccade target in the nonswitch trials: for the switch trials the saccade target was in the direction in which switch neurons exhibited switch-selective activity (e.g., the contralateral target for contra-switch neurons), and for the nonswitch trials the saccade target was in the opposite direction (e.g., the ipsilateral target for contra-switch neurons). The rationale for this comparison is as follows: the monkey's set before target onset should be the same between these two kinds of trials (e.g., primed for the ipsilateral target) and any switch-selective activity must appear as a divergence of activity in the switch trials from activity in the nonswitch trials. In this comparison, we included all of the nonswitch trials regardless of their numerical position in each block (see Fig. $2 A, n-2, n-1, n+1, n+2$ ). This is based on the following reasons. First, the occurrence of the switch trial was unpredictable to the monkeys and therefore it was impossible for the animals to specify the position of the current nonswitch trial relative to the upcoming switch trial. Note that even an $(n+1)$ nonswitch trial and an $(n+2)$ nonswitch trial could become an $(n-1)$ nonswitch trial if the number of trials in a given block is 1 and 2, respectively. Second, the animal's motor processes, including the motor set before cue onset and saccade preparation/execution process after cue onset, should be the same regardless of the position of nonswitch trials. Third, the SRTs and error rate were, indeed, not significantly different across nonswitch trials (see Fig. 2 A). Finally, presaccadic activity of switch neurons was nondifferential between nonswitch trials (see Figs. 3A, $4 A$ ). In estimating the neuronal differentiation time described above, we adopted the following conservative procedure: we chose the nonswitch trials whose SRTs were longer than the behavioral differentiation time in the saccade overriding task ( $\geq 223 \mathrm{~ms}$ for monkey T; $\geq 233 \mathrm{~ms}$ for monkey S). Because the SRTs in the nonswitch trials were consistently shorter (Fig. 2A), inclusion of all nonswitch trials would make the neuronal differentiation time unfairly shorter. Second, we used an asymmetric kernel that resembled a postsynaptic potential to construct SDFs for this precise time course analysis (Thompson et al., 1996). Motivation for the use of this asymmetric kernel is that, with this kernel, each spike exerts influence only forward in time, whereas with the Gaussian kernel, spikes also exert influence backward in time.

The neuronal differentiation time for the population of switch neurons (see Fig. 5A) was also estimated using resampling. For example, as described in Table 1, we recorded from 38 increase-type switch-selective activities (we analyzed separately each direction of switch-selective activity for bilateral switch neurons). This means that we had 38 pairs of SDFs (constructed with the asymmetric kernel) for the switch and nonswitch trials. From these, we drew 38 pairs of activities with random replacement and constructed the ensemble-averaged activity for the switch and nonswitch trials. We then sought the time at which the two ensembleaveraged activities started to differentiate. For this purpose, we first searched for the time bin (bin width, $1 \mathrm{~ms}$ ) where the difference in the two activities was maximum, during an interval delimited by the cue onset and the longest SRT. We then sought backward in time for the first time bin where the two activities first crossed ("intersection time"). To estimate the confidence limits for the intersection time, we repeated this procedure 1000 times and took the 97.5th percentile point as the neuronal differentiation time for the population of neurons.

All of the statistical procedures were assessed by two-tailed tests and performed using commercial software (Matlab 7.0; MathWorks).

\section{Results}

\section{Switching from automatic to controlled eye movement}

A critical feature of the saccade overriding task (Fig. 1A) was that the color of the cue remained the same in a block of varying number of trials. Because of this feature, it seemed natural for the monkey to start preparing for a saccade before the cue was actu- 
Table 1. Classification of switch neurons

\begin{tabular}{|c|c|c|c|c|c|c|c|c|}
\hline & \multicolumn{2}{|c|}{ Contra-switch neurons } & \multicolumn{2}{|c|}{ Ipsi-switch neurons } & \multicolumn{4}{|c|}{ Bilateral-switch neurons } \\
\hline & Inc & Dec & $\operatorname{lnc}$ & Dec & $\begin{array}{l}\text { Contra-Inc and } \\
\text { Ipsi-Inc }\end{array}$ & $\begin{array}{l}\text { Contra-Dec and } \\
\text { Ipsi-Dec }\end{array}$ & $\begin{array}{l}\text { Contra-Inc and } \\
\text { Ipsi-Dec }\end{array}$ & $\begin{array}{l}\text { Contra-Dec } \\
\text { and Ipsi-Inc }\end{array}$ \\
\hline Contralateral go & 3 & & & & & & 2 & \\
\hline Ipsilateral no-go & 7 & & & 4 & 1 & & 4 & \\
\hline $\begin{array}{l}\text { Contralateral go and Ipsilateral no-go } \\
\text { Ipsilateral go }\end{array}$ & 9 & & & & & & 2 & \\
\hline Contralateral no-go & & & 1 & & & & & 1 \\
\hline Ipsilateral go and Contralateral no-go & & & 1 & & & & & \\
\hline Bilateral go & & & & & & & & \\
\hline Bilateral no-go & 3 & & & & & & & \\
\hline Nonselective & 1 & & & 1 & 1 & & & \\
\hline Not tested & 1 & & & 1 & & & & \\
\hline
\end{tabular}

Values denote the number of neurons. Inc, Increase type; Dec, decrease type.

ally given, by assuming that the color of the upcoming cue would be the same as that in the previous trial. A short time interval between the stimulus onset and cue onset could also facilitate such an anticipatory motor preparation and generation. Indeed, saccade behavior of the animal was different between the switch trials and nonswitch trials. The saccadic reaction times (SRTs), measured from the cue onset, were significantly shorter and error rates were reliably lower in the nonswitch trials ( $p<0.001$, post hoc Tukey's test) (Fig. 2A), indicating that the saccade behavior was more automatic or habitual in the nonswitch trials. However, such automatic processing suddenly became inappropriate in the switch trials because the habitual saccade was based on the preceding color rule and therefore was bound to be wrong in the current color rule (Fig. $2 B$, gray). For successful saccade switching, the monkey had to suppress an automatic saccade that was no longer valid and instead facilitate an alternative saccade by following the cue instruction. These additional processes led to a substantial switch cost (Monsell, 2003): significantly longer SRTs and higher error rates on the switch trials (Fig. $2 A$ ).

A comparison of SRT distributions between the correct switch trials and the incorrect switch trials provided another important piece of information on this behavior. The animal's performance on the switch trials became above the chance level at $223 \mathrm{~ms}$ from cue onset for monkey $\mathrm{T}$ and $233 \mathrm{~ms}$ for monkey $\mathrm{S}(p<0.05$, binomial test) (Fig. $2 B$ ), which, hereafter, we call behavioral differentiation time. This temporal information is useful to consider whether neural activity can control the switch. Supposing there are neurons somewhere in the brain that are capable of switching in the saccade overriding task, then their activity must differentiate between the switch trials and nonswitch trials before the behavioral differentiation time.

B
A Saccade overriding task $\quad$ Switch trial

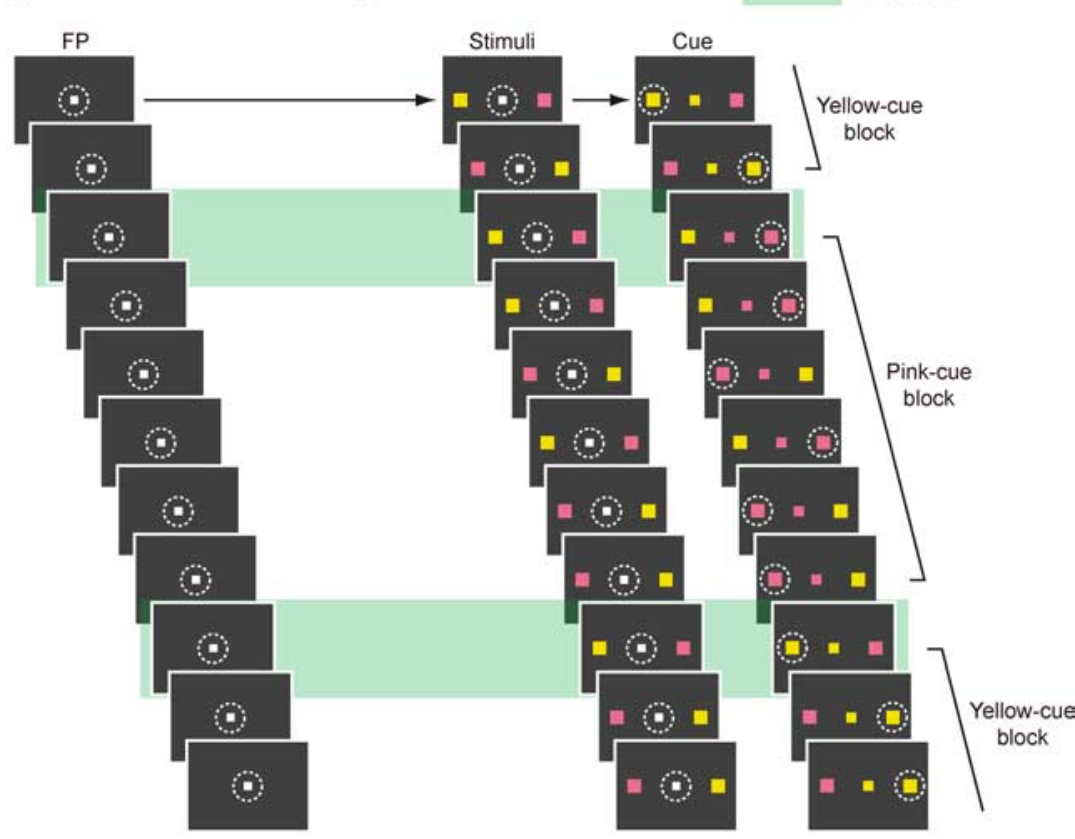
Saccade Go/Nogo task

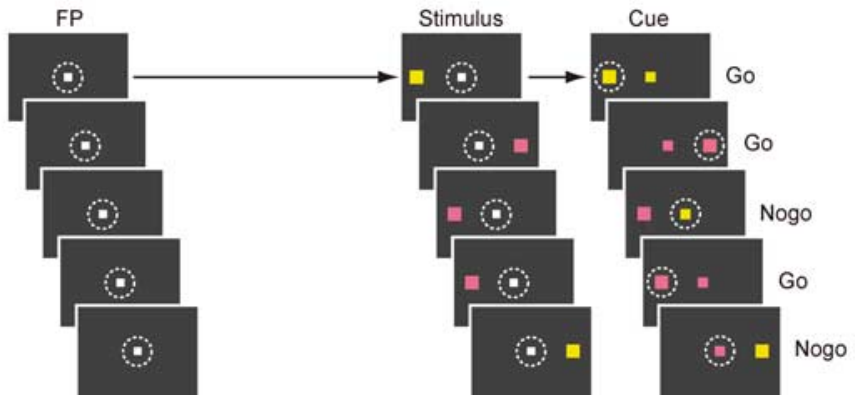

Figure 1. Behavioral tasks. $\boldsymbol{A}$, The sequence of events in the saccade overriding task. White dotted circles (invisible to the monkey in actual experiments) indicate the direction of gaze required for the correct responses. Green rectangles indicate switch trials. Note that in the switch trials, the monkey had to switch the saccade from a primed stimulus that had been the target to a nonprimed stimulus that would become the target. $\boldsymbol{B}$, The sequence of events in the saccade go/no-go task. White dotted circles (invisible to the monkey in actual experiment) indicate the direction of gaze for the correct responses.

\section{Switch-selective neuronal activity in the STN}

We studied the activity of 145 single neurons in the STN as the animal performed the saccade overriding task. Of these, presaccadic activity of 43 neurons (29.7\%) differed significantly in the 
A

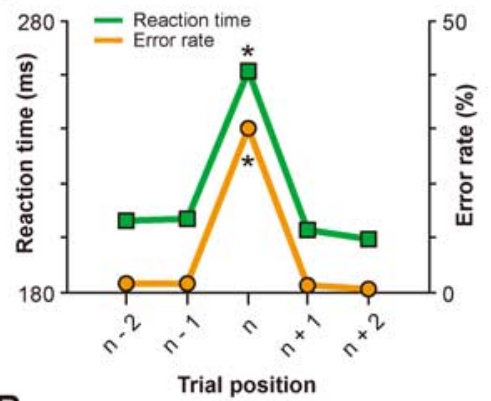

B

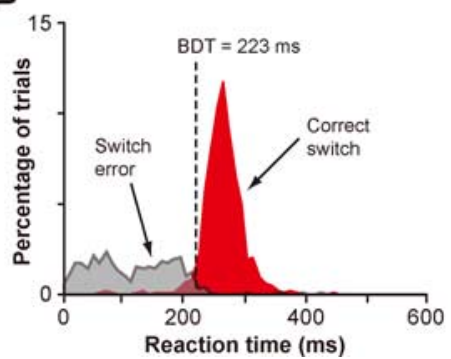

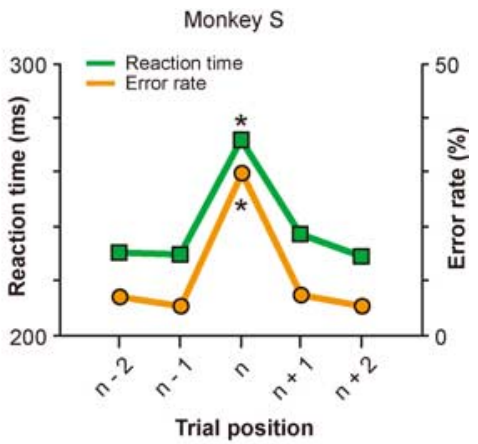

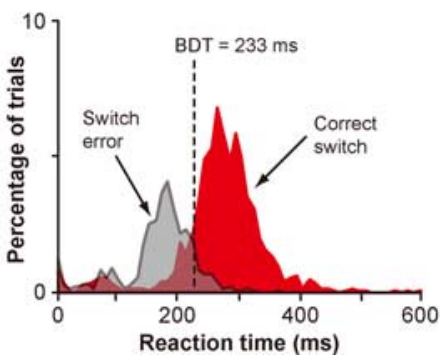

Figure 2. Animal performance in the saccade overriding task. $\boldsymbol{A}$, Average SRTs (green) and average error rates (orange) plotted against the trial position in each block ( $n$ represents the switch trials) for monkey $T$ (left) and monkey $S$ (right). ${ }^{*} p<0.001$. $\boldsymbol{B}$ Distribution of SRTs for the correct switch trials (red) and incorrect switch trials (gray) for monkey T (left; $n=1426$ for correct switches and 711 for switch errors) and monkey $S$ (right; $n=1532$ for correct switches and 739 for switch errors). Black broken lines represent the behavioral differentiation time (BDT).

switch trials ("switch neurons") (for a statistical definition, see Materials and Methods, Data analysis). Most switch neurons were selective for the direction of the switch. Figure $3 A$ shows an example of switch neurons in the STN. This neuron exhibited a significant increase in activity before saccade onset in the switch trials when the saccade was to be directed to the contralateral side. We refer to this neuron as a contra-switch neuron because it was activated when the animal switched from the primed ipsilateral nontarget to the nonprimed contralateral target. Figure $4 \mathrm{~A}$ shows another example of switch neurons, in which switch-selective presaccadic activity was observed when the saccade was made to the ipsilateral side. We refer to this neuron as an ipsi-switch neuron. Contra-switch neurons outnumbered ipsi-switch neurons in population (Table 1). Like these examples, the majority of switch neurons increased their activity in the switch trials (increase type; $n=28$ ). Some switch neurons decreased their activity in the switch trials (decrease type; $n=6$ ), whereas the remaining neurons showed a switch-selective increase for one saccade direction and a switch-selective decrease for the opposite direction $(n=9)$ (Table 1).

We considered whether the switch-selective activity was influenced by the color of the cue. Among a total of 54 switch-selective activities (bilateral-switch neurons have two switch-selective activities), 49 (91\%) were nondifferential between the two cue colors $(p>0.05, t$ test). We also considered whether the switchselective activity was different depending on a change in the saccade direction from the previous trial to the current switch trial. We found that 53 switch-selective activities (98\%) were not influenced by whether the saccade direction in the switch trial was the same as, or opposite to, that in the previous nonswitch trial $(p>0.05, t$ test). These data suggest that the activity of switch neurons is concerned with switching from habitual to controlled saccades rather than switching the direction of the upcoming saccade or switching of the particular color of the cue.

To calculate the time at which the switch-selective activity

emerged in response to the cue switch, we aligned the ensemble-averaged activity of switch neurons on the cue onset (Fig. 5A) and compared it between the correct switch trials (red) and correct nonswitch trials (blue), separately for the increase type (left) and the decrease type (right). Not surprisingly, the activities in the switch trials and nonswitch trials were almost identical initially. This is likely because in both cases the monkey was primed for the stimulus that had been the target in the nonswitch trials. However, the two sets of activity diverged at $174 \mathrm{~ms}$ after the cue onset for the increase type ( $p<0.05$, resampling) (Fig. 5A, left). We will refer to this time as neuronal differentiation time. Importantly, the neuronal differentiation time was well in advance of the behavioral differentiation time (Fig. $2 B$ ), suggesting that the switch-selective activity for the increase-type switch neurons started early enough to enable the switch. Although the neuronal differentiation time for the decrease-type switch neurons was much later (214 ms after the cue onset) (Fig. 5A, right), it was still earlier than the behavioral differentiation time. However, when the monkey failed to switch the saccade (gray), neuronal differentiation occurred at $247 \mathrm{~ms}$ for the increase type and $292 \mathrm{~ms}$ for the decrease type, both of which were later than the behavioral differentiation time. Therefore, the timing of onset of switch-selective activity appears to be important for the animal to successfully switch the saccade.

Figure 6 illustrates the spatial distribution of switch neurons in the right STN for monkey T. In this figure, the locations of switch neurons (red circles) and nonswitch neurons (black circles) are plotted based on the coronal histological sections (Fig. $6 A)$. We found that switch neurons and nonswitch neurons were intermingled with each other and that switch neurons were distributed mainly in the ventral half of the STN (Fig. 6B).

\section{Characterization of individual switch-selective neurons}

The switch-selective activity may be interpreted in two different ways: (1) it suppresses the unwanted saccade that is triggered by the habitual process, or (2) it facilitates the desired saccade that is based on the controlled process. To distinguish between these possibilities, we further characterized the activity of individual switch neurons using the saccade go/no-go task (Fig. $1 B$ ). Figure $3 B$ illustrates the activity of the contra-switch neuron shown in Figure $3 A$ but now as the animal performed the saccade go/no-go task. When the stimulus appeared on the contralateral side, the activity was nondifferential between go and no-go trials $(p>$ 0.05 , resampling) (Fig. $3 B$, left). When the stimulus was presented on the ipsilateral side, however, the activity of this neuron was significantly larger in no-go trials than go trials $(p<0.05$, resampling) (Fig. $3 B$, right). There was an increase in activity which started slightly before cue onset, but this activation was unrelated to the go/no-go decision because it occurred in both go trials and no-go trials. The ipsilateral no-go activation suggests that the action of this neuron would be to inhibit ipsiversive saccades. This hypothetical action was consistent with the requirement for this neuron to switch contralaterally in the saccade 
A Saccade overriding task Contralateral target

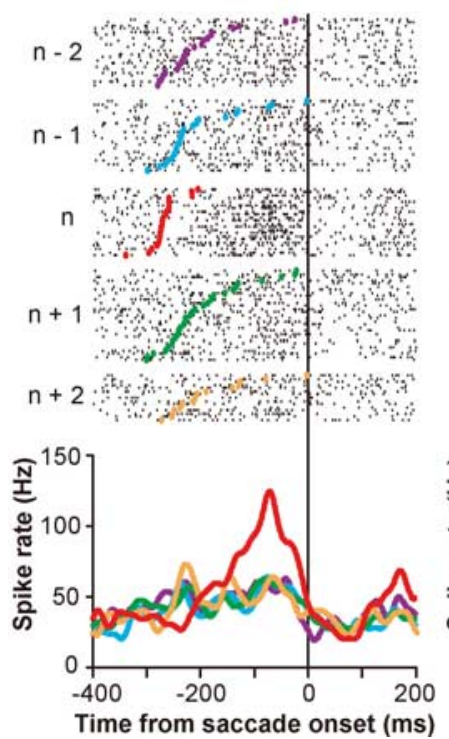

Ipsilateral target
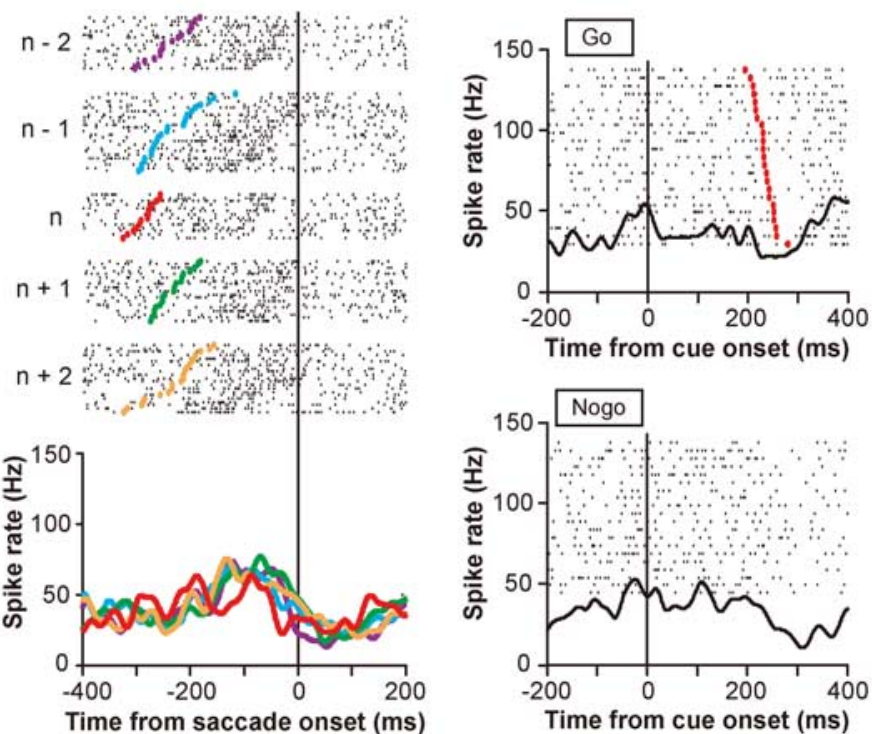

B Saccade Go/Nogo task Contralateral target

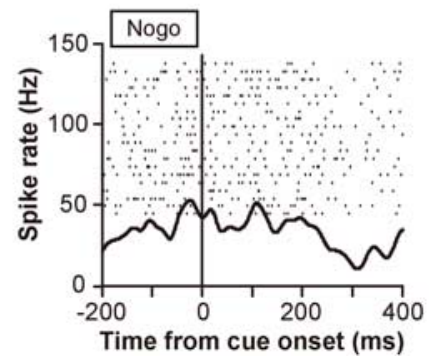

Ipsilateral target
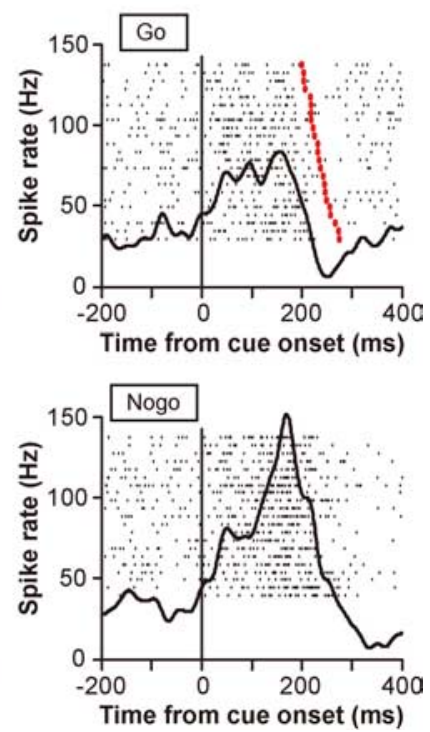

Figure 3. An example of a contra-switch neuron (no-go type). $\boldsymbol{A}$, Switch-selective activity in the correctly performed saccade overriding task. Rastergrams and SDFs are sorted by the trial position in each block ( $n$ represents the switch trials). In the left column, the saccade target was on the contralateral side for both switch trials $(n)$ and nonswitch trials $(n-2, n-1, n+1, n+2)$; in the right column, the saccade target was on the ipsilateral side for both switch trials and nonswitch trials. Rastergrams and SDFs are aligned with saccade onset. In the rastergrams, small black dots represent the time of individual action potentials and colored dots indicate the time of cue onset; trials are arranged in order of SRTs. $\boldsymbol{B}$, Ipsilateral no-go activity in the saccade go/no-go task. Rastergrams and SDFs are grouped by the type of trials (go or no-go trials) and the direction of the target. Rastergrams and SDFs are aligned with cue onset. In go trials (top), red dots indicate the time of saccade onset and trials are arranged in order of SRTs.

\section{A Saccade overriding task} Contralateral target
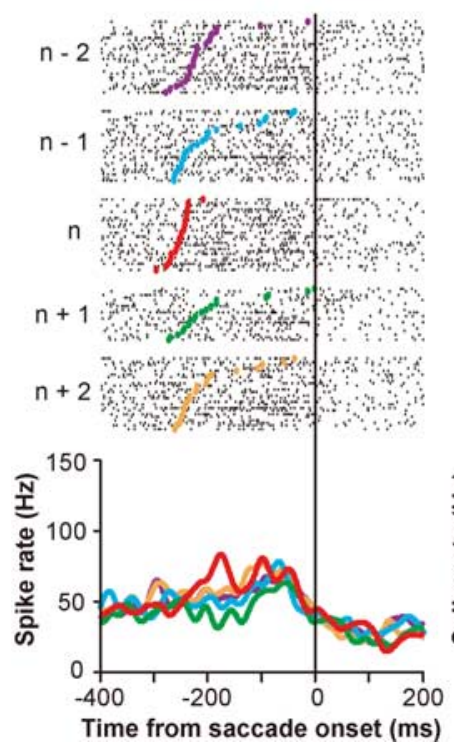

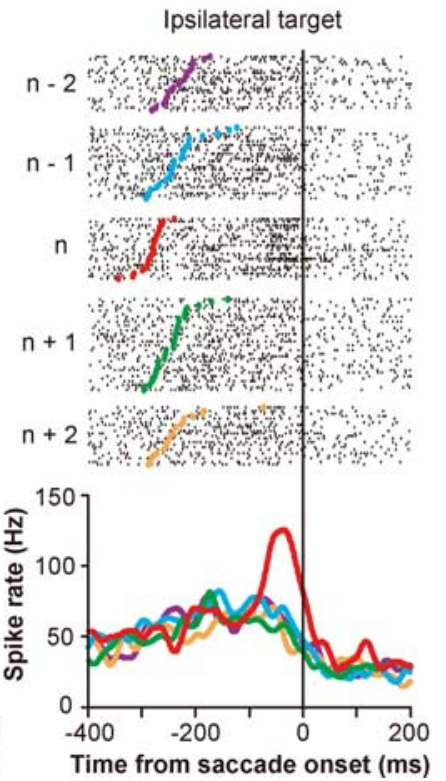

B

Saccade Go/Nogo task

Contralateral target
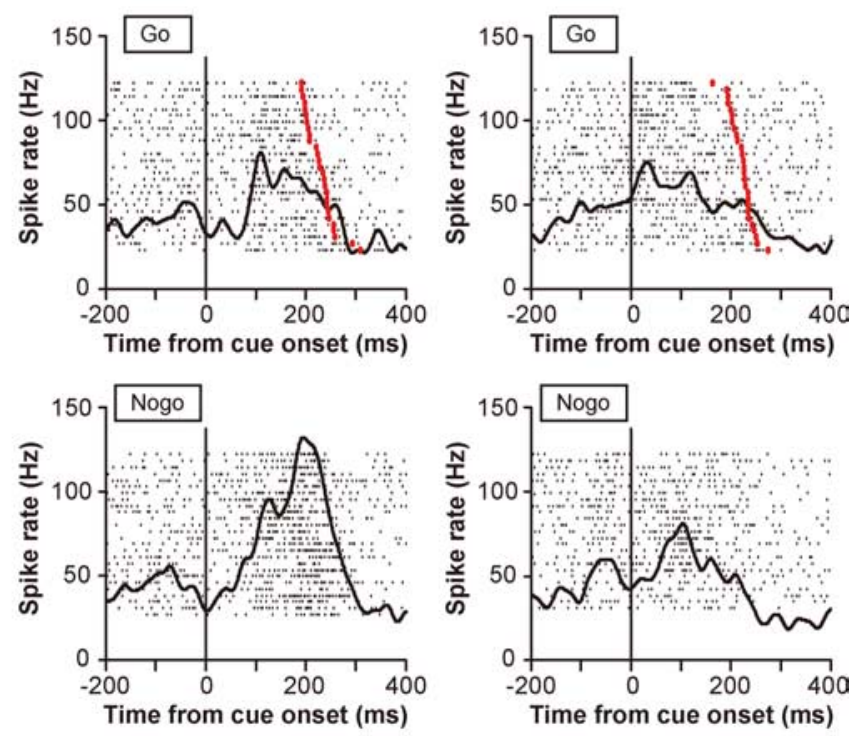

Figure 4. An example of an ipsi-switch neuron (no-go type). $\boldsymbol{A}$, Switch-selective activity in the correctly performed saccade overriding task (same conventions as in Fig. $3 A$ ). $\boldsymbol{B}$, Contralateral no-go activity in the saccade go/no-go task (same conventions as in Fig. 3B).

overriding task. However, the function of the ipsi-switch neuron shown in Figure $4 A$ was found to inhibit contraversive saccades (Fig. $4 B$ ). Note again that this neuronal action (contralateral nogo) accords with the requirement for this neuron to switch ipsilaterally. We classified these examples of switch neurons as the no-go type.

The use of the saccade go/no-go task allowed us to classify switch neurons into four functional types: suppression only (no-go type), facilitation only (go type), both suppression and facilitation (dual type) (for an example of activity, see supplemental Fig. 2, available at www.jneurosci.org as supplemental material), and no apparent selectivity (NS type). Among 41 switch neurons that were also tested in the saccade go/no-go task, the majority were the no-go type $(n=21)$ (Fig. $5 D$, left). The dual type was the second most common $(n=12)$, whereas the go type was in the minority $(n=5)$ (Fig. $5 D$, left). This is in marked 
A

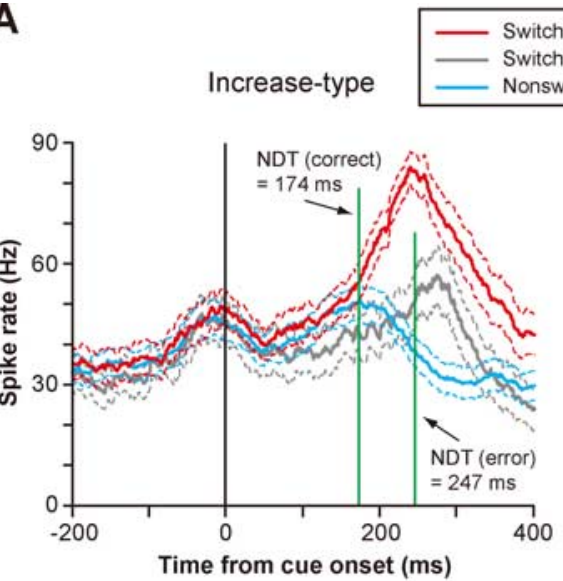

B

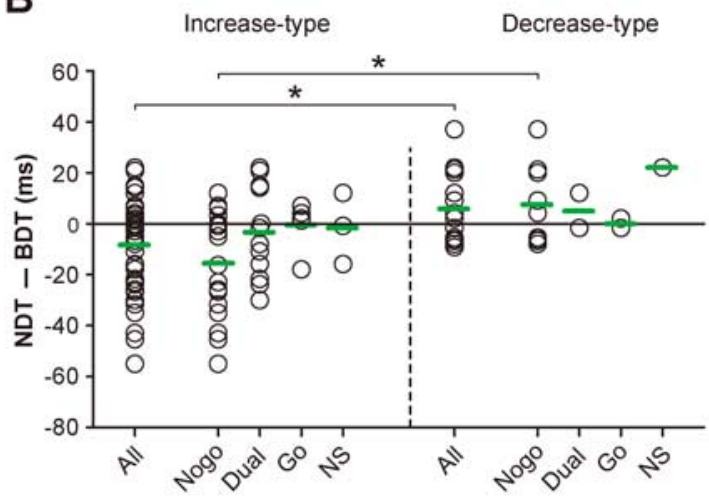

\section{Decrease-type}

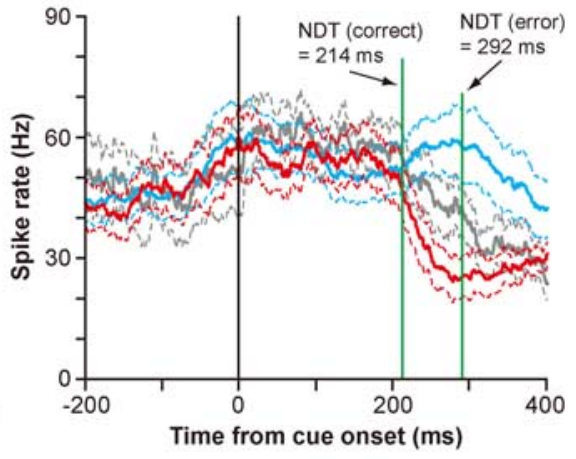

C

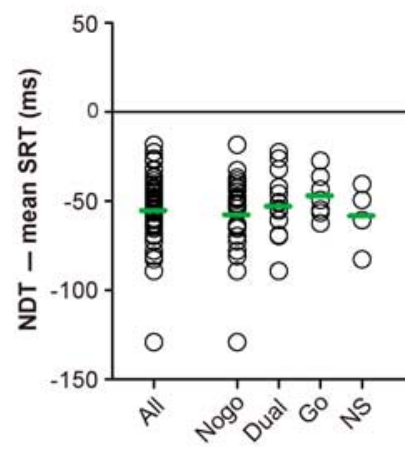

D

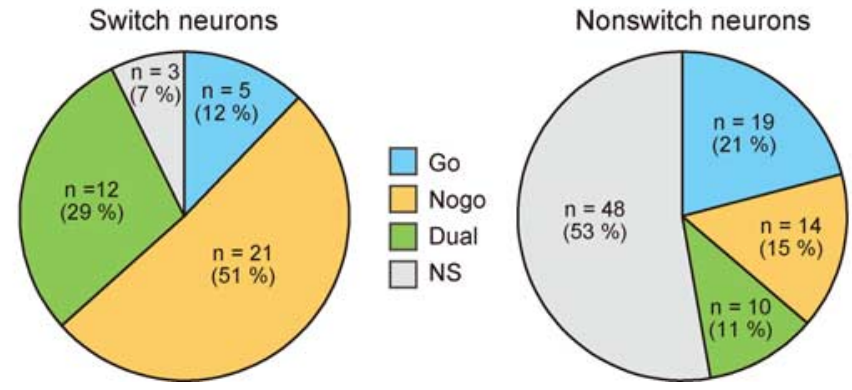

Figure 5. Population summary of switch-selective activity. $\boldsymbol{A}$, The neuronal differentiation time (NDT) for the population of switch-selective activity. Ensemble-averaged SDFs (mean \pm SD) for the increase type (left) and the decrease type (right) in the correct switch trials (red), incorrect switch trials (gray), and correct nonswitch trials (blue) are shown. SDFs are aligned with cue onset. Note that, for this comparison, the direction of the saccade target in the switch trials is opposite that in the nonswitch trials (see Materials and Methods). $\boldsymbol{B}$, The neuronal differentiation time of individual switch-selective activity for the increase type (left) and decrease type (right). Plotted along the ordinate is the neuronal differentiation time relative to the behavioral differentiation time. Negative values indicate that neuronal differentiation occurred before behavioral differentiation. Switchselective activity is grouped by its functional subtype. Green horizontal arrows represent the mean. Bilateral-switch neurons yielded two data points. ${ }^{*} p<0.01$. C, Comparison of the neuronal differentiation time with the mean SRT obtained from each session. The increase type and decrease type are combined together. Negative values indicate that the neuronal differentiation time preceded the average SRT. Green horizontal arrows represent the group mean. $\boldsymbol{D}$, Frequency distribution of functional subtypes for switch neurons (left) and nonswitch neurons (right).

contrast to the distribution of functional types in nonswitch neurons $\left(p<0.001, \chi^{2}\right.$ test), in which the NS type was the most prevalent and the go type was the second most common (Fig. $5 D$, right). This finding suggests that no-go-type and dual-type switch neurons were preferentially used for behavioral switching. Importantly, the activity pattern of most switch neurons was compatible with a role in the inhibition of unwanted and/or the enhancement of wanted saccades (Table 1). These data suggest that the switch-selective activity is the control signal for saccade switching that is mainly used for the suppression of habitual unwanted saccades or both the suppression of habitual saccades and the facilitation of controlled alternative saccades.

It was tempting to know whether the switch-selective activity of individual switch neurons started early enough to enable the switch. We thus measured the neuronal differentiation time for each switch neuron and compared it with the behavioral differentiation time. We found that $58 \%$ (22 of 38 ) and $43 \%$ (6 of 14) of the neuronal differentiation time preceded the behavioral differentiation time for the increase type and the decrease type, respectively (Fig. 5B). The neuronal differentiation time was significantly earlier for the increase type than for the decrease type $(p<0.01, t$ test) (Fig. 5B). Among a total of 28 switch-selective activities whose neuronal differentiation time was earlier than the behavioral differentiation time, 16 were no-go type, 8 were dual type, 2 were go type, and 2 were NS type. Although $46 \%$ (24 of 52) of the neuronal differentiation time was later than the behavioral differentiation time (Fig. $5 B$ ), all of them were still earlier than the average SRT (Fig. 5C), suggesting that the switch-selective activity begins early enough to influence saccade behavior.

\section{Discussion}

Here, we report that successful switching from a habitual saccade to a controlled saccade was associated with a significant increase, or occasional decrease, in presaccadic activity for a group of STN neurons. This switch-selective activity was direction selective and usually observed when the saccade was switched to the contralateral side. Importantly, the neuronal differentiation time for the population of switch neurons was earlier than the behavioral differentiation time. A further test using the saccade go/no-go task suggested that the majority of switch neurons inhibited an automatic undesired saccade while some facilitated a controlled desired saccade; some did both. These actions operated in a spatially selective manner, which accorded with the direction of switching.

It seems unlikely that the switchselective activity reflects a neuronal process related to an increased level of arousal because arousal should not depend on the direction of switching. However, it could reflect a shift of attention or a direction-selective conflict between automatic and controlled saccades. However, we have no additional evidence to resolve this issue. In the following discussion we will instead focus on the possible mechanisms with which STN neurons might participate in switching from automatic saccades to controlled saccades. 
In the present study and our previous study (Isoda and Hikosaka, 2007) we show that both the STN and pre-SMA contain switch neurons. The next question is, then, How could the switch-selective activity differ between the pre-SMA and STN? Because we used the same experimental protocol in the same animals, we can directly compare neural activity between the two structures. We found two major differences. First, no-go-type, go-type, and dual-type switch neurons were almost equally common in the pre-SMA whereas the majority of switch neurons in the STN were the no-go type ( $p<0.01, \chi^{2}$ test) (supplemental Fig. 3, available at www. jneurosci.org as supplemental material). This suggests that the pre-SMA is involved in the inhibition of automatic action as well as the facilitation of controlled action, both of which are necessary processes for behavioral switching (Monsell, 2003). In contrast, the STN appears to be more specialized for the inhibition of automatic action. Second, the neuronal differentiation time was earlier in the pre-SMA than in the STN (supplemental Fig. 4, available at www.jneurosci.org as supplemental mate-

rial); their time difference was $16 \mathrm{~ms}$. This finding is consistent with the hypothesis that the switch signal in the STN originates from the pre-SMA rather than the STN influencing the pre-SMA through output nuclei of the basal ganglia and the thalamus (Alexander et al., 1986). The anatomical finding is also consistent with this hypothesis: the locations of switch neurons in the STN were mainly in its ventral part (Fig. 6B), which roughly correspond to the pre-SMA projection area (Inase et al., 1999). Given that STN neurons have long dendrites that can extend nearly two-thirds of the structure (Sato et al., 2000), it seems possible that STN switch neurons receive afferents directly from pre-SMA switch neurons. Together, these data support our hypothesis that the pre-SMA may use the STN to implement its switching function.

This conclusion resembles what has been shown by functional neuroimaging studies (Aron and Poldrack, 2006; Aron et al., 2007) but extends understanding of STN functions in several ways. First, we showed the STN mechanism of behavioral switching at the single cell level. Second, a precise time course analysis of neuronal activity suggested a causal relationship of the switch signals from the pre-SMA to the STN. Third, the switch signal in the STN begins early enough to influence the saccade behavior. We showed that the neuronal differentiation time for all of the STN switch neurons was earlier than the average saccade latency. This temporal relationship holds even if the average efferent conduction delay for STN neurons $(\sim 12 \mathrm{~ms})$ is subtracted: $1.1 \mathrm{~ms}$ from the STN to the substantia nigra pars reticulata (SNr) (estimated by antidromic activation) (Kita et al., 1983), 1.3-1.5 ms from the SNr to the superior colliculus (SC) (estimated by antidromic activation) (Hikosaka and Wurtz, 1983; Jiang et al., 2003), $8.0 \mathrm{~ms}$ from the SC to saccade outputs (estimated by orthodromic activation) (Miyashita and Hikosaka, 1996), and 0.3-0.4 ms for each synaptic transmission (Eccles, 1964). Fourth, the use of the saccade go/no-go task allowed us to characterize the function of individual switch neurons. Specifically, we found many no-go-type neurons as suggested by clinical observations, but we also found, quite unexpectedly, the presence of dual-type and go-type neurons. Finally, such no-go and go actions of STN switch neurons were mostly spatially selective.

How, then, is it possible for the STN to exert its inhibitory and facilitatory effects on saccadic motor output in a spatially selective manner? Figure 7 illustrates hypothetical circuit diagrams that can account for the underlying neuronal pathways. We assume that the no-go pathway consists of a direct connection from the STN to the SNr, which in turn projects to the SC (Fig. 7A). Because STN neurons are excitatory and SNr neurons are inhibitory, activation of STN neurons should result in the suppression of SC output neurons. The suppression of ipsiversive saccades can be mediated by $\mathrm{SNr}$ neurons projecting to the $\mathrm{SC}$ on the opposite side (Fig. 7A, left) (Beckstead et al., 1981; Jiang et al., 2003), whereas contraversive suppression can be mediated by SNr neurons projecting to the SC on the same side (Beckstead et al., 1981; Hikosaka and Wurtz, 1983) (Fig. 7A, right). However, we postulate that the go pathway uses indirect connections from the STN to the SNr through the external segment of the globus pallidus (GPe) (Fig. 7B). In support of this idea, it has been shown that electrical stimulation in the motor cortex produces "early" excitation in GPe neurons via the STN (Nambu et al., 2000; Kita et al., 2004). Because GPe neurons are inhibitory, the net effect of the STN output would be reversed from no-go to go. Again, directional selectivity of go action can be explained by whether SNr neurons connect to the SC on the opposite side (ipsilateral go) (Fig. $7 B$, left) or on the same side (contralateral go) (Fig. $7 B$, right).

Note, however, that individual switch neurons in the STN often had a combination of actions (Table 1). For example, the most common type was contra-switch neurons with contra-go and ipsi-no-go actions. According to the scheme in Figure 7, these neurons would have efferent connections both to the $\mathrm{SNr}$ and the GPe. Indeed, a single axon tracing study in cynomolgus 
A Nogo pathway

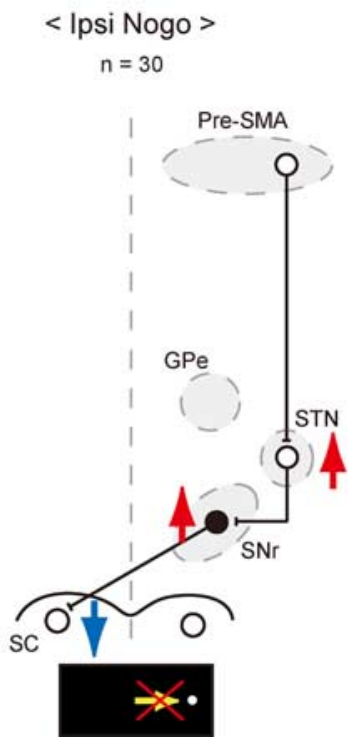

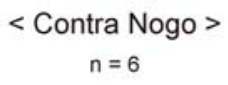

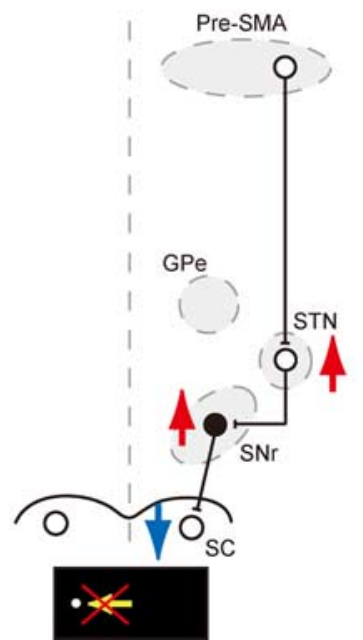

B Go pathway

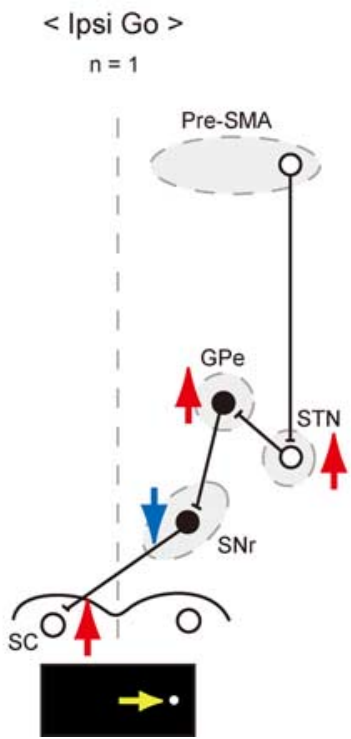

$<$ Contra Go >
$n=16$

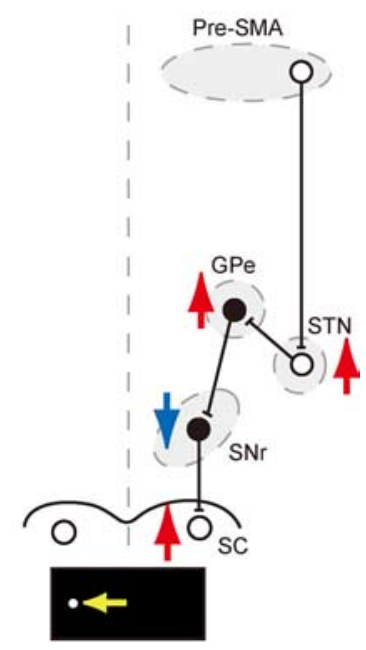

Figure 7. Hypothetical neural circuits for direction-selective no-go and go functions. A, Hypothetical no-go pathways. Red upward arrows indicate excitation of neuronal activity, whereas blue downward arrows indicate inhibition of neuronal activity. Yellow horizontal arrows in the bottom represent a saccade to be suppressed. Shown are the number of switch neurons having each neuronal action. Open circles depict excitatory neurons, whereas black filled circles show inhibitory neurons. $\boldsymbol{B}$, Hypothetical go pathways (same conventions as in $\boldsymbol{A}$ ). Yellow horizontal arrows in the bottom represent a saccade to be facilitated.

monkeys showed that STN neurons projecting to the SNr also send collaterals to the GPe, or to both the GPe and GPi (Parent et al., 2000; Sato et al., 2000). However, it is not clear why our data suggest the existence of STN switch neurons with exclusive connections to the $\mathrm{SNr}$ (i.e., with only no-go actions), whereas the anatomical study did not find such SNr-only connections. There may be several possibilities to explain this discrepancy. First, switch neurons may be a unique group of neurons in the STN, some of which have SNr-only connections. Because switch neurons composed $\sim 30 \%$ of all sampled neurons, the likelihood of encountering the neurons with SNr-only connections in single axon tracing studies may be low. Second, even if an STN neuron has connections to both the $\mathrm{SNr}$ and the GPe, their synaptic effects may not be strong enough to produce observable changes in firing rate in our tasks. Third, some of the no-go type neurons may have connections to the GPe, in addition to the connections to the SNr (Fig. 7), but the recipient GPe neurons send back inhibitory connections to the STN to terminate the switchselective activity. Fourth, some of the efferent connections may have nonoculomotor effects if the recipient neurons in the SNr or the GPe have connections to nonoculomotor areas. The last possibility is interesting because behavioral switching is usually not a local motor phenomenon but requires several different processes such as the switch of mental operations (e.g., cognitive strategies or task implementations), enhancement of sensory responsiveness, cessation of a set of ongoing bodily movements and orienting responses, and facilitation of a desired set of motor activities. It is then possible that axon collaterals to the nonoculomotor neurons in the GPe and GPi underlie these diverse aspects of behavioral switching.

According to our schema in Figure 7, the connections between the STN and the GPe, and their connections to the SNr, provide many options for the cerebral frontal cortex to implement quick control of behavior. The function of a particular cortical cell would be determined by downstream projection patterns of an
STN neuron with which this cortical cell connects. Thus, what a cortical input needs to do is simply to pick one or a combination of them. Such multiple options might be an outcome of unsupervised, random connections between neurons in the STN, SNr, and GPe. However, cortical neurons (e.g., pre-SMA neurons) need to be connected appropriately to particular combinations of random connections. At this point, there is no definitive answer to the question of how the right connection might be chosen. One possibility is that it is achieved by reinforcement learning mechanisms. Dopaminergic innervations of STN neurons (Hassani et al., 1997; Prensa et al., 2000) might underlie such a possible plastic mechanism.

In conclusion, the STN and pre-SMA play important roles in switching from automatic behavior to deliberately controlled behavior. Our data suggest that the switch signal in the pre-SMA is implemented by the STN, consistent with the idea that the cortico-subthalamic pathway is ideal for quick implementation of behavioral control requiring a cessation of ongoing movement. Whether the switching function in these areas extends beyond the motor domain, such as thoughts and emotions, is an interesting question for future research.

\section{References}

Alexander GE, DeLong MR, Strick PL (1986) Parallel organization of functionally segregated circuits linking basal ganglia and cortex. Annu Rev Neurosci 9:357-381.

Aron AR, Poldrack RA (2006) Cortical and subcortical contributions to Stop signal response inhibition: role of the subthalamic nucleus. J Neurosci 26:2424-2433.

Aron AR, Behrens TE, Smith S, Frank MJ, Poldrack RA (2007) Triangulating a cognitive control network using diffusion-weighted magnetic resonance imaging (MRI) and functional MRI. J Neurosci 27:3743-3752.

Baunez C, Nieoullon A, Amalric M (1995) In a rat model of parkinsonism, lesions of the subthalamic nucleus reverse increases of reaction time but induce a dramatic premature responding deficit. J Neurosci 15:6531-6541.

Bauswein E, Fromm C, Preuss A (1989) Corticostriatal cells in comparison 
with pyramidal tract neurons: contrasting properties in the behaving monkey. Brain Res 493:198-203.

Beckstead RM, Edwards SB, Frankfurter A (1981) A comparison of the intranigral distribution of nigrotectal neurons labeled with horseradish peroxidase in the monkey, cat, and rat. J Neurosci 1:121-125.

Bergman H, Wichmann T, DeLong MR (1990) Reversal of experimental parkinsonism by lesions of the subthalamic nucleus. Science 249:1436-1438.

Bergman H, Wichmann T, Karmon B, DeLong MR (1994) The primate subthalamic nucleus. II. Neuronal activity in the MPTP model of parkinsonism. J Neurophysiol 72:507-520.

Crist CF, Yamasaki DS, Komatsu H, Wurtz RH (1988) A grid system and a microsyringe for single cell recording. J Neurosci Methods 26:117-122.

Crone EA, Wendelken C, Donohue SE, Bunge SA (2006) Neural evidence for dissociable components of task-switching. Cereb Cortex 16:475-486.

Crossman AR, Sambrook MA, Jackson A (1984) Experimental hemichorea/ hemiballismus in the monkey. Studies on the intracerebral site of action in a drug-induced dyskinesia. Brain 107:579-596.

DeLong MR (1990) Primate models of movement disorders of basal ganglia origin. Trends Neurosci 13:281-285.

DeLong MR, Crutcher MD, Georgopoulos AP (1985) Primate globus pallidus and subthalamic nucleus: functional organization. J Neurophysiol 53:530-543.

Eccles JC (1964) Physiology of synapses. Berlin: Springer.

Efron B, Tibshirani RJ (1993) An introduction to the Bootstrap. Boca Raton, Florida: Chapman and Hall/CRC.

Frank MJ, Samanta J, Moustafa AA, Sherman SJ (2007) Hold your horses: impulsivity, deep brain stimulation, and medication in parkinsonism. Science 318:1309-1312.

Hassani OK, François C, Yelnik J, Féger J (1997) Evidence for a dopaminergic innervation of the subthalamic nucleus in the rat. Brain Res 749:88-94.

Hikosaka O, Wurtz RH (1983) Visual and oculomotor functions of monkey substantia nigra pars reticulata. IV. Relation of substantia nigra to superior colliculus. J Neurophysiol 49:1285-1301.

Hikosaka O, Sakamoto M, Miyashita N (1993) Effects of caudate nucleus stimulation on substantia nigra cell activity in monkey. Exp Brain Res 95:457-472.

Inase M, Tokuno H, Nambu A, Akazawa T, Takada M (1999) Corticostriatal and corticosubthalamic input zones from the presupplementary motor area in the macaque monkey: comparison with the input zones from the supplementary motor area. Brain Res 833:191-201.

Isoda M, Hikosaka O (2007) Switching from automatic to controlled action by monkey medial frontal cortex. Nat Neurosci 10:240-248.

Jiang H, Stein BE, McHaffie JG (2003) Opposing basal ganglia processes shape midbrain visuomotor activity bilaterally. Nature 423:982-986.

Judge SJ, Richmond BJ, Chu FC (1980) Implantation of magnetic search coils for measurement of eye position: an improved method. Vision Res 20:535-538.

Kita H, Chang HT, Kitai ST (1983) Pallidal inputs to subthalamus: intracellular analysis. Brain Res 264:255-265.

Kita H, Nambu A, Kaneda K, Tachibana Y, Takada M (2004) Role of ionotropic glutamatergic and GABAergic inputs on the firing activity of neurons in the external pallidum in awake monkeys. J Neurophysiol 92:3069-3084.

Kusama T, Mabuchi M (1970) Stereotaxic atlas of the brain of Macaca fuscata. Tokyo: University of Tokyo.

Limousin P, Pollak P, Benazzouz A, Hoffmann D, Le Bas JF, Broussolle E, Perret JE, Benabid AL (1995) Effect of parkinsonian signs and symptoms of bilateral subthalamic nucleus stimulation. Lancet 345:91-95.

Matsumura M, Kojima J, Gardiner TW, Hikosaka O (1992) Visual and oculomotor functions of monkey subthalamic nucleus. J Neurophysiol 67:1615-1632.
Mink JW (1996) The basal ganglia: focused selection and inhibition of competing motor programs. Prog Neurobiol 50:381-425.

Miyashita N, Hikosaka O (1996) Minimal synaptic delay in the saccadic output pathway of the superior colliculus studied in awake monkey. Exp Brain Res 112:187-196.

Monakow K, Akert K, Künzle H (1978) Projections of the precentral motor cortex and other cortical areas of the frontal lobe to the subthalamic nucleus in the monkey. Exp Brain Res 33:395-403.

Monchi O, Petrides M, Strafella AP, Worsley KJ, Doyon J (2006) Functional role of the basal ganglia in the planning and execution of actions. Ann Neurol 59:257-264.

Monsell S (2003) Task switching. Trends Cogn Sci 7:134-140.

Nachev P, Rees G, Parton A, Kennard C, Husain M (2005) Volition and conflict in human medial frontal cortex. Curr Biol 15:122-128.

Nachev P, Wydell H, O’Neill K, Husain M, Kennard C (2007) The role of the pre-supplementary motor area in the control of action. Neuroimage 36 [Suppl 2]:T155-T163.

Nakamura K, Sakai K, Hikosaka O (1998) Neuronal activity in medial frontal cortex during learning of sequential procedures. J Neurophysiol 80:2671-2687.

Nambu A, Takada M, Inase M, Tokuno H (1996) Dual somatotopical representations in the primate subthalamic nucleus: evidence for ordered but reversed body-map transformations from the primary motor cortex and the supplementary motor area. J Neurosci 16:2671-2683.

Nambu A, Tokuno H, Hamada I, Kita H, Imanishi M, Akazawa T, Ikeuchi Y, Hasegawa N (2000) Excitatory cortical inputs to pallidal neurons via the subthalamic nucleus in the monkey. J Neurophysiol 84:289-300.

Nambu A, Tokuno H, Takada M (2002) Functional significance of the cortico-subthalamo-pallidal "hyperdirect" pathway. Neurosci Res 43:111-117.

Norman DA, Shallice T (1986) Attention to action: willed and automatic control of behavior. In: Consciousness and self regulation: advances in research and theory (Davidson R, Schwartz G, Shapiro D, eds), pp 1-18. New York: Plenum.

Parent A, Sato F, Wu Y, Gauthier J, Lévesque M, Parent M (2000) Organization of the basal ganglia: the importance of axonal collateralization. Trends Neurosci 23:S20-S27.

Prensa L, Cossette M, Parent A (2000) Dopaminergic innervation of human basal ganglia. J Chem Neuroanat 20:207-213.

Robinson DA (1963) A method of measuring eye movement using a scleral search coil in a magnetic field. IEEE Trans Biomed Eng 10:137-145.

Rushworth MF, Hadland KA, Paus T, Sipila PK (2002) Role of the human medial frontal cortex in task switching: a combined fMRI and TMS study. J Neurophysiol 87:2577-2592.

Sato F, Parent M, Levesque M, Parent A (2000) Axonal branching pattern of neurons of the subthalamic nucleus in primates. J Comp Neurol 424:142-152.

Shima K, Mushiake H, Saito N, Tanji J (1996) Role for cells in the presupplementary motor area in updating motor plans. Proc Natl Acad Sci U S A 93:8694-8698.

Thompson KG, Hanes DP, Bichot NP, Schall JD (1996) Perceptual and motor processing stages identified in the activity of macaque frontal eye field neurons during visual search. J Neurophysiol 76:4040-4055.

Wichmann T, Bergman H, DeLong MR (1994) The primate subthalamic nucleus. I. Functional properties in intact animals. J Neurophysiol 72:494-506.

Woodward TS, Ruff CC, Ngan ET (2006) Short- and long-term changes in anterior cingulate activation during resolution of task-set competition. Brain Res 1068:161-169.

Yoshida M, Precht W (1971) Monosynaptic inhibition of neurons of the substantia nigra by caudato-nigral fibers. Brain Res 32:225-228. 